\title{
Sur les périodes des nombres quadratiques spécialisés d'une fonction algébrique quadratique "réelle"
}

\author{
par \\ R. Paysant-Le Roux (Caen)
}

I. Introduction et résultat principal. Dans deux articles A. Schinzel ([11]) étudie la longueur des périodes des fractions continues des nombres quadratiques réels $\sqrt{F(n)}$ où $n \in \mathbb{Z}$ et $F(n)$ est un polynôme non carré de la forme

$$
a_{0}^{2} n^{2 p+2}+a_{1} n^{2 p+1}+\ldots+a_{2 p+2},
$$

$p, a_{i}$ entiers, $p \geq 0, a_{0} \neq 0$.

Pour cela, il introduit la fraction continue formelle de la série formelle

$$
\begin{aligned}
\sqrt{F(X)} & =a_{0} X^{p+1}\left(1+\frac{a_{1}}{a_{0}^{2}} \frac{1}{X}+\ldots+\frac{a_{2 p+2}}{a_{0}^{2}} \frac{1}{X^{2 p+2}}\right)^{1 / 2} \\
& =a_{0} X^{p+1}\left(1+\frac{a_{1}}{2 a_{0}^{2}} \frac{1}{X}+\ldots\right) \in \mathbb{Q}((1 / X)) .
\end{aligned}
$$

Si cette fraction continue est périodique de longueur $\pi$, il pose $\operatorname{LP} \sqrt{F}=\pi$, si elle n'est pas périodique il pose $\operatorname{LP} \sqrt{F}=\infty$.

Il définit également les numérateurs et les dénominateurs des réduites de cette fraction continue :

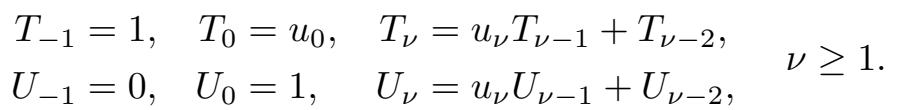

Enfin, il note par $\operatorname{lp} \sqrt{F(n)}$ la longueur de la fraction continue du nombre quadratique réel $\sqrt{F(n)}$ si $F(n)$ est positif et n'est pas un carré parfait. En notant par $\widetilde{\mathbb{Z}}$ l'ensemble des entiers $n$ de $\mathbb{Z}$ tels que $F(n)>0$ et non carré parfait, il obtient le théorème suivant :

ThÉorème 1 (A. Schinzel). 1. Si LP $\sqrt{F}=\infty$ alors

$$
\lim _{\substack{n \rightarrow \infty \\ n \in \mathbb{Z}}} \operatorname{lp} \sqrt{F(n)}=\infty \text {. }
$$


2. Si $\operatorname{LP} \sqrt{F}=\pi>0$ et $\sqrt{F}=\left[u_{0}, \overline{u_{1}, \ldots, u_{\pi}}\right]$, notons par $E$ l'ensemble des entiers $n \in \widetilde{\mathbb{Z}}$ tels que $2 T_{\pi-1}(n)$ est entier, et par $C E$ son complémentaire. Alors :

(i) $\varlimsup_{\substack{n \rightarrow \infty \\ n \in E}} \operatorname{lp} \sqrt{F(n)}<\infty$,

(ii) $\lim _{\substack{n \rightarrow \infty \\ n \in C E}} \operatorname{lp} \sqrt{F(n)}=\infty$.

Nous étudions ici le problème similaire au précédent, à savoir :

On se donne un polynôme $F$ de la forme (1) et sans racine multiple; on peut alors considérer le corps de fonctions $K_{X}=\mathbb{Q}(X, \sqrt{F})$; enfin, on se donne une fonction $f \in K_{X} \backslash \mathbb{Q}(X)$. On étudie alors la période du nombre quadratique réel $f(n)$ lorsque $n$ décrit $\widetilde{\mathbb{Z}}$.

Notre étude va montrer que c'est la notion de quasi-périodicité (périodicité à une constante multiplicative près) du développement en fraction continue de la fonction $f$ qui est essentielle ici. Dans le cas où le développement est quasi-périodique, on sait [6] que cette propriété est équivalente au fait que l'anneau des stabilisateurs du $\mathbb{Q}[X]$-module $\mathcal{M}_{X}:=\mathbb{Q}[X]+f \mathbb{Q}[X]$ admet une unité non triviale; si on note par $\mathcal{E}$ une unité fondamentale du groupe des unités de norme \pm 1 de cet anneau, on a alors la généralisation du théorème de A. Schinzel.

1. Si le développement en fraction continue formel de $f(X)$ n'est pas quasi-périodique alors la longueur de la période du nombre quadratique réel $f(n)$ tend vers l'infini avec $n, n \in \widetilde{\mathbb{Z}}$.

2. Si le développement en fraction continue formel de $f(X)$ est quasipériodique alors notons par $\mathcal{E}(X)$ l'unité fondamentale du groupe des unités de norme \pm 1 de l'anneau des stabilisateurs du module $\mathcal{M}_{X}$, et par $E$ l'ensemble des entiers de $\widetilde{\mathbb{Z}}$ tels que $\mathcal{E}(n)$ soit une unité du corps quadratique réel $\mathbb{Q}(\sqrt{F(n)})$. On a :

(i) $\varlimsup_{\substack{n \rightarrow \infty \\ n \in E}} \operatorname{lp} f(n)<\infty$,

(ii) $\lim _{\substack{n \rightarrow \infty \\ n \in \mathbb{Z} \backslash E}} \operatorname{lp} f(n)=\infty$.

II. Fraction continue formelle de $f(X)$

A. Développement en fraction continue formelle de $f(X) \in K_{X} \backslash \mathbb{Q}(X)$. On considère $f$ comme un élément de $\mathbb{Q}((1 / X))$ et on pose $f=E(f)+1 / f_{1}$ avec $E(f) \in \mathbb{Q}[X]$ et $f_{1} \in \mathbb{Q}((1 / X)),\left|f_{1}\right|>1$, où $\left|f_{1}\right|=e^{\operatorname{deg} f_{1}}, \operatorname{deg} f_{1}=m_{0}$ si $f_{1}=\sum_{m=m_{0}}^{\infty} c_{m} X^{-m}, c_{m_{0}} \neq 0$. De même, on pose pour tout $\nu \geq 0$,

$$
f_{\nu}=E\left(f_{\nu}\right)+\frac{1}{f_{\nu+1}}, \quad E\left(f_{\nu}\right) \in \mathbb{Q}[X],\left|f_{\nu+1}\right|>1 .
$$


On obtient alors ce qu'on appelle le développement en fraction continue formelle de $f(X)$. On peut alors définir la suite des réduites $\left(P_{\nu} / Q_{\nu}\right)_{\nu \geq 0} \mathrm{du}$ développement.

Les polynômes $P_{\nu}$ et $Q_{\nu}$ sont définis par les égalités

$$
\begin{array}{llll}
P_{-2}=0, & P_{-1}=1, & P_{\nu}=E\left(f_{\nu}\right) P_{\nu-1}+P_{\nu-2}, & \nu \geq 0 \\
Q_{-2}=1, & Q_{-1}=0, & Q_{\nu}=E\left(f_{\nu}\right) Q_{\nu-1}+Q_{\nu-2}, & \nu \geq 0 .
\end{array}
$$

On a

on peut donc écrire

$$
\lim _{\nu \rightarrow \infty} P_{\nu} / Q_{\nu}=f
$$

$$
f=\left[E(f), E\left(f_{1}\right), \ldots, E\left(f_{\nu}\right), \ldots\right]
$$

où $E\left(f_{\nu}\right) \in \mathbb{Q}[X]$ et $\operatorname{deg} E\left(f_{\nu}\right) \geq 1$, pour tout $\nu \geq 1$.

B. Caractérisation des éléments $f \in K_{X} \backslash \mathbb{Q}(X)$ périodique (resp. quasipériodique). Considérons la fraction continue formelle de $f$ :

$$
f(X)=\left[u_{0}(X), u_{1}(X), \ldots\right], \quad u_{i} \in \mathbb{Q}[X], \operatorname{deg} u_{i} \geq 1, i \geq 1 .
$$

On sait qu'il est infini car $f \notin \mathbb{Q}(X)$. On a $f_{i}=\left[u_{i}(X), u_{i+1}(X), \ldots\right] \in$ $K_{X}, i \geq 0$. Nous pouvons alors définir un développement périodique et un développement quasi-périodique (voir [1], [2], [6]) :

DÉFinitions (périodicité et quasi-périodicité). Soit $f \in K_{X} \backslash \mathbb{Q}(X)$. Le développement en fraction continue de $f$ est dit périodique (resp. quasipériodique) s'il existe deux entiers $i \geq 0, \pi \geq 1$ tels que pour tout entier $j \geq i$ on a $f_{j+\pi}=f_{j}$ (resp. pour tout entier $j \geq i$ il existe un rationnel non nul $c_{j}$ tel que $f_{j+\pi}=c_{j} f_{j}$ ).

Re marques. 1. Si $f$ est quasi-périodique alors on peut définir la quasipériode $\pi^{*}(f)$ comme étant le plus petit entier $\geq 1$ vérifiant la propriété. Il est alors facile de voir que si $\pi^{*}(f)$ est pair l'ensemble des constantes $c_{j}$ se réduit à l'ensemble $\left\{c^{-1}, c\right\}$ pour un certain $c \in \mathbb{Q}^{*}$. Nous dirons dans ce cas que $c$ (ou $c^{-1}$ ) est la constante attachée au développement de $f$.

2. L'exemple

$$
f:=\frac{4 X^{2}+1+\sqrt{16 X^{4}+24 X^{2}+1}}{8 X}=\left[c_{0} X, c_{1} X, \ldots\right]
$$

où

$$
c_{i}= \begin{cases}2^{i / 2} & \text { pour } i \text { pair, } \\ 2^{(3-i) / 2} & \text { pour } i \text { impair, }\end{cases}
$$

montre qu'il existe des fonctions quasi-périodiques qui ne sont pas périodiques.

Pour caractériser ces développements nous allons introduire quelques objets et notations. 
Considérons le $\mathbb{Q}[X]$-module $\mathcal{M}_{f}=\mathbb{Q}[X]+f \mathbb{Q}[X]$ et son anneau des stabilisateurs $\mathcal{O}_{f}=\left\{\gamma \in K_{X}: \gamma \mathcal{M}_{f} \subset \mathcal{M}_{f}\right\}$. On sait que c'est un $\mathbb{Q}[X]$ module de rang 2 et qu'il est inclus dans l'anneau des $\mathbb{Q}[X]$-entiers de $K_{X}$. L'anneau des $\mathbb{Q}[X]$-entiers est $\mathbb{Q}[X, \sqrt{F}]$ car $F$ n'a pas de racine multiple. Notons $\mathcal{U}_{f}$ le groupe des unités de $\mathcal{O}_{f}$. On a évidemment $\mathbb{Q}^{*} \subset \mathcal{U}_{f}$. Enfin, $\mathcal{U}_{f}^{(1)}$ désignera le sous-groupe des unités de $\mathcal{O}_{f}$ de norme \pm 1 .

On a l'injection canonique

$$
\mathcal{U}_{f}^{(1)} /\{-1,1\} \hookrightarrow \mathcal{U}_{f} / \mathbb{Q}^{*}
$$

On pose

$$
G_{f}=\mathcal{U}_{f} / \mathbb{Q}^{*}, \quad G_{f}^{(1)}=\mathcal{U}_{f}^{(1)} /\{-1,1\} .
$$

La caractérisation que nous donnons ici résulte de nombreux travaux anciens ou plus récents (voir [1], [2], [5], [6]).

ThÉORÈme 2. Soit $f \in K_{X} \backslash \mathbb{Q}(X)$. La fonction $f$ est quasi-périodique si et seulement si $\mathbb{Q}^{*} \subsetneq \mathcal{U}_{f}$. La fonction $f$ est périodique si et seulement si, soit $f$ est quasi-périodique et $\pi^{*}(f)$ est impair, soit $f$ est quasi-périodique, $\pi^{*}(f)$ est pair et la constante $c \in \mathbb{Q}^{*}$ attachée au développement est \pm 1 .

EXemples. 1. Soit $f=(L+\sqrt{F}) / M, L, M \in \mathbb{Q}[X], M \neq 0$ et $M \mid L^{2}-F$. On a dans ce cas $\mathcal{O}_{f}=\mathbb{Q}[X, \sqrt{F}]$ et donc les équivalences suivantes (voir Berry [2]) :

$$
\begin{aligned}
f \text { est quasi-périodique } & \Leftrightarrow \mathbb{Q}^{*} \subsetneq \mathcal{U}_{f}=\mathcal{U}_{\sqrt{F}} \\
& \Leftrightarrow \sqrt{F} \text { est quasi-périodique } \\
& \Leftrightarrow \sqrt{F} \text { est périodique. }
\end{aligned}
$$

2. Soit $f=(X-\alpha) \sqrt{X^{2}+h}, h \neq 0$. On a ici $\mathcal{O}_{f}=\mathbb{Q}[X]+f \mathbb{Q}[X]$ et le résultat suivant (voir A. Schinzel [11]) :

$$
f \text { quasi-périodique } \Leftrightarrow \alpha=0 \text { ou } h=-\frac{4}{3} \alpha^{2}, 2 \alpha^{2} \text { ou } 4 \alpha^{2} .
$$

\section{Spécialisation}

Notation. Soit $f \in K_{X} \backslash \mathbb{Q}(X)$. Désignons par LQP $(f)$ la longueur de la plus courte quasi-période de $f$ si $f$ est quasi-périodique et l'infini sinon.

On désigne par $\widetilde{\mathbb{Z}}$ l'ensemble des entiers $n \in \mathbb{Z}$ tels que $F(n)$ soit positif et non carré parfait, et par $K_{n}$ le corps quadratique réel $\mathbb{Q}(\sqrt{F(n)})$ pour $n \in \widetilde{\mathbb{Z}}$.

Enfin, on désigne par lp $f(n)$ la longueur de la période du développement en fraction continue de $f(n)$.

On a alors que la nature formelle du développement influence fortement le développement en fraction continue du quadratique réel $f(n)$ (si celui-ci est défini). 
THÉORÈme 3 (théorème principal). 1. Si $\mathrm{LPQ}(f)=\infty$ alors

$$
\lim _{\substack{n \rightarrow \infty \\ n \in \mathbb{Z}}} \operatorname{lp} f(n)=\infty
$$

2. Si $\operatorname{LPQ}(f)<\infty$.

Désignons par $\mathcal{E}(X)$ l'unité fondamentale du sous-groupe des unités de norme \pm 1 de $\mathcal{O}_{f}$, et par $E$ l'ensemble des entiers $n \in \widetilde{\mathbb{Z}}$ tels que $\mathcal{E}(n)$ soit un entier du corps quadratique réel $\mathbb{Q}(\sqrt{F(n)})$. Alors on $a$ :

(i) $\varlimsup_{\substack{n \rightarrow \infty \\ n \in E}} \operatorname{lp} f(n)<\infty$,

(ii) $\lim _{\substack{n \rightarrow \infty \\ n \notin E}} \operatorname{lp} f(n)=\infty$.

\section{Points extrémaux et faces d'un module}

Notations. Soit $n \in \widetilde{\mathbb{Z}}$.

- $\mathcal{M}_{X}\left(\right.$ resp. $\left.\mathcal{M}_{n}\right)$ désigne le $\mathbb{Q}[X]$ - (resp. $\mathbb{Z}$-) module $\mathbb{Q}[X]+f(X) \mathbb{Q}[X]$ (resp. $\mathbb{Z}+f(n) \mathbb{Z})$.

- $\mathcal{O}_{X}$ (resp. $\mathcal{O}_{n}$ ) désigne l'anneau des stabilisateurs du module $\mathcal{M}_{X}$ $\left(\right.$ resp. $\left.\mathcal{M}_{n}\right)$.

- $\mathcal{U}_{X}$ (resp. $\mathcal{U}_{n}$ ) désigne le groupe des unités modulo $\mathbb{Q}^{*}$ (resp. modulo $\pm 1)$ de l'anneau $\mathcal{O}_{X}\left(\operatorname{resp} . \mathcal{O}_{n}\right)$.

- $\mathcal{U}_{X}^{(1)}$ désigne le groupe des unités de norme \pm 1 de l'anneau $\mathcal{O}_{X}$; ces unités sont définies au signe près.

- On note par ||$_{1}$ et ||$_{2}$ les deux valeurs absolues définies sur $K_{X}$ prolongeant la valeur absolue à l'infini définie sur $\mathbb{Q}(X)$ par $|P|=e^{\operatorname{deg} P}$ si $P \in \mathbb{Q}[X] \backslash\{0\}$.

Si $\alpha(X) \in K_{X} \backslash\{0\}$ alors $\alpha(X)$ peut s'écrire

$$
\alpha(X)=\sum_{m=m_{0}}^{\infty} c_{m} X^{-m}, \quad c_{m_{0}} \neq 0 .
$$

On pose $|\alpha(X)|_{1}=e^{m_{0}}$ et $|\alpha(X)|_{2}=|\bar{\alpha}(X)|_{1}$ où $\bar{\alpha}$ désigne le conjugué de $\alpha$ dans $K_{X}$.

DÉFINITION D'Un POINT EXTRÉMAL. On appelle point extrémal du module $\mathcal{M}_{X}\left(\operatorname{resp} . \mathcal{M}_{n}\right)$ un élément $\varphi$ non nul de $\mathcal{M}_{X}\left(\operatorname{resp} . \mathcal{M}_{n}\right)$ possédant la propriété :

$$
\begin{gathered}
\left(\forall \psi \in \mathcal{M}_{X} \backslash\{0\},|\psi|_{i} \leq|\varphi|_{i}, \forall i=1,2\right) \Rightarrow\left(\exists \lambda \in \mathbb{Q}^{*}, \psi=\lambda \varphi\right) \\
\left.\quad \text { (resp. } \forall \psi \in \mathcal{M}_{n} \backslash\{0\},|\psi|_{i} \leq|\varphi|_{i}, \forall i=1,2 \Rightarrow \psi= \pm \varphi\right) .
\end{gathered}
$$


DÉFinition D'Une FACE. On appelle face $d u$ module $\mathcal{M}_{X}$ un élément $\varphi$ non nul de ce module vérifiant

$$
\left(\forall \psi \in \mathcal{M} \backslash\{0\},|\psi|_{i} \leq|\varphi|_{i}, \forall i=1,2\right) \Rightarrow\left(\exists i,|\psi|_{i}=|\varphi|_{i}\right) .
$$

On note par $\mathcal{E}_{X}\left(\operatorname{resp} . \mathcal{E}_{n}\right)$ l'ensemble des points extrémaux modulo $\mathbb{Q}^{*}$ (resp. $\bmod \pm 1)$ de $\mathcal{M}_{X}\left(\right.$ resp. $\left.\mathcal{M}_{n}\right)$.

Cet ensemble $\mathcal{E}_{X}$ (resp. $\mathcal{E}_{n}$ ) est muni d'une structure de graphe grâce à la notion de voisinage.

DÉfinition De points extrémaux voisins. Deux points extrémaux $\varphi$ et $\psi$ du module $\mathcal{M}_{X}\left(\right.$ resp. $\left.\mathcal{M}_{n}\right)$ sont dits voisins si

$$
\left(\forall \pi \in \mathcal{M}_{X}\left(\operatorname{resp} . \mathcal{M}_{n}\right),|\pi|_{i}<\max \left\{|\psi|_{i},|\varphi|_{i}\right\}, \forall i=1,2\right) \Rightarrow \pi=0 .
$$

La structure de ce graphe est une double chaîne infinie, totalement ordonnée par les valeurs absolues 1 ou 2 :

$$
\begin{aligned}
& \begin{array}{llllll}
\ldots \varphi_{-2} & \varphi_{-1} & \varphi_{0} & \varphi_{1} & \varphi_{2} & \ldots
\end{array} \\
& \ldots>\left|\varphi_{-2}\right|_{1}>\left|\varphi_{-1}\right|_{1}>\left|\varphi_{0}\right|_{1}>\left|\varphi_{1}\right|_{1}>\left|\varphi_{2}\right|_{1}>\ldots, \\
& \ldots<\left|\varphi_{-2}\right|_{2}<\left|\varphi_{-1}\right|_{2}<\left|\varphi_{0}\right|_{2}<\left|\varphi_{1}\right|_{2}<\left|\varphi_{2}\right|_{2}<\ldots \text {, }
\end{aligned}
$$

où $\varphi_{0}$ est défini de la façon suivante :

$$
\left|\varphi_{0}\right|=\min \left\{|\varphi|_{2}: \varphi \text { point extrémal et }|\varphi|_{2} \geq 1\right\} .
$$

La suite $\left(\varphi_{k}\right)_{k \geq 0}$ est appelée suite des points extrémaux de $\mathcal{M}_{X}$ (resp. $\left.\mathcal{M}_{n}\right)$ dans la direction ||$_{2}$.

On peut définir l'ensemble quotient $\mathcal{E}_{X} / \mathcal{U}_{X}\left(\right.$ resp. $\left.\mathcal{E}_{n} / \mathcal{U}_{n}\right)$ et $\mathcal{E}_{X} / \mathcal{U}_{X}^{(1)}$ d'une structure de graphe en remarquant que les groupes $\mathcal{U}_{X}$ (resp. $\mathcal{U}_{n}$ ) et $\mathcal{U}_{X}^{(1)}$ opèrent $\operatorname{sur} \mathcal{E}_{X}\left(\operatorname{resp} . \mathcal{E}_{n}\right)$ et que la notion de voisinage passe au quotient.

Structure de ces graphes quotients

1. Si $\mathcal{U}_{X}$ est trivial (le groupe des unités est réduit à $\mathbb{Q}^{*}$ ) alors $\mathcal{U}_{X}^{(1)}$ est aussi trivial et le graphe $\mathcal{E}_{X} / \mathcal{U}_{X}$ est le même que celui de $\mathcal{E}_{X}$.

2. Si $\mathcal{U}_{X}$ est non trivial alors $\mathcal{U}_{X}^{(1)}$ est non trivial et les graphes $\mathcal{E}_{X} / \mathcal{U}_{X}$ et $\mathcal{E}_{X} / \mathcal{U}_{X}^{(1)}$ sont des cycles qui sont les mêmes si $\mathcal{U}_{X}^{(1)}=\mathcal{U}_{X}$ et l'un a le double d'éléments de l'autre si $\mathcal{U}_{X}^{(1)} \subsetneq \mathcal{U}_{X}$ : 

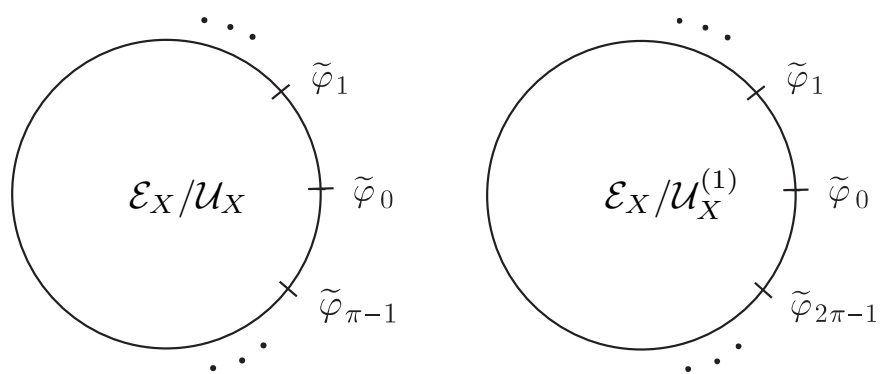

$$
\left|\mathcal{E}_{X} / \mathcal{U}_{X}^{(1)}\right|=\left\{\begin{array}{ll}
\left|\mathcal{E}_{X} / \mathcal{U}_{X}\right| & \text { si } \mathcal{U}_{X}^{(1)}=\mathcal{U}_{X}, \\
2\left|\mathcal{E}_{X} / \mathcal{U}_{X}\right| & \text { si } \mathcal{U}_{X}^{(1)} \subsetneq \mathcal{U}_{X},
\end{array} \quad\left[G_{X}: G_{X}^{(1)}\right]=2 .\right.
$$

Enfin, le graphe $\mathcal{E}_{n} / \mathcal{U}_{n}$ est un cycle.

\section{Unités. Points extrémaux. Faces et fraction continue}

1. Unités. Points extrémaux et fraction continue

\section{DÉFINITIONS}

- Soient $n \in \widetilde{\mathbb{Z}}$ et $f \in K_{X}$ (resp. $f(n) \in K_{n}$ ). On dit que l'élément $f$ est réduit s'il vérifie les inégalités

$$
|f|_{1}>1(\text { resp. } f(n)>1) \text { et }|f|_{2}<1(\text { resp. }-1<\bar{f}(n)<0) \text {. }
$$

- Soit $f \in K_{X}$ (resp. $f(n) \in K_{n}$ ). Nous dirons qu'il est presque réduit s'il vérifie $|f|_{1}>1$ et $|f|_{2} \leq 1$ (resp. $f(n)>1$ et $\bar{f}(n)<0$ ).

Désignons par $\left(P_{k} / Q_{k}\right)_{k \geq-1}$ (resp. $\left.\widetilde{P}_{k} / \widetilde{Q}_{k}\right)$ la suite du développement en fraction continue de $f$ (resp. $f(n)$ ). On a alors le résultat suivant :

Proposition 1. Soit $f \in K_{X}$ (resp. $f(n) \in K_{n}$ ) un élément presque réduit. Alors :

(i) La suite des points extrémaux $\left(\varphi_{k}\right)_{k \geq 0}\left(\right.$ resp. $\left.\left(\widetilde{\varphi}_{k}\right)_{k \geq 0}\right)$ de $\mathcal{M}_{X}$ (resp. $\left.\mathcal{M}_{n}\right)$ dans la direction ||$_{2}$ est donnée par

$$
\begin{gathered}
\varphi_{k+1}=P_{k}-Q_{k} f, \quad k \geq-1 \\
\left(\text { resp. } \widetilde{\varphi}_{k+1}=\widetilde{P}_{k}-\widetilde{Q}_{k} f(n), \quad k \geq-1\right) .
\end{gathered}
$$

(ii) Dans le cas formel, si $f$ admet un développement en fraction continue quasi-périodique de longueur de quasi-période $\pi$ alors les unités de l'anneau des stabilisateurs du module $\mathcal{M}_{X}$ dans la direction ||$_{2}$ sont données, modulo $\mathbb{Q}^{*}$, par

$$
\varphi_{k \pi}=P_{k \pi-1}-Q_{k \pi-1} f, \quad k \geq 0 .
$$


Dans le cas réel si nous désignons par $\pi$ la longueur de la période du développement en fraction continue de $f(n)$, alors les unités de l'anneau des stabilisateurs sont données, modulo \pm 1 , par

$$
\varphi_{k \pi}=P_{k \pi-1}-Q_{k \pi-1} f(n), \quad k \geq 0 .
$$

Preuve. Dans le cas formel elle résulte de la proposition 7 de [6] en remarquant que cette proposition reste vraie si on remplace l'hypothèse " $f$ réduit" par " $f$ presque réduit". Dans le cas réel voir [7], et dans des cas particuliers déjà [9], tome I, théorèmes 3.18 et 3.35 .

\section{Fraction continue régulière de $f(n)$}

Proposition 2. Pour tout $\nu \in \mathbb{N}^{*}$, il existe une partition des entiers de $\widetilde{\mathbb{Z}}$ en un nombre fini de classes infinies $E_{1}, \ldots, E_{s}$ telles que pour tout $n \in E_{i}, n$ assez grand, le début du développement en fraction continue de $f(n)$ s'écrit

$$
f(n)=\left[b_{0}(n),\left[\left[\frac{d_{0}}{j_{0}}\right]\right], \ldots, b_{\nu-1}(n),\left[\left[\frac{d_{\nu-1}}{j_{\nu-1}}\right]\right], \beta_{\nu}\right]
$$

où :

1. $d_{l}, j_{l}, 0 \leq l<\nu$, sont des entiers positifs ou nuls indépendant de l'entier $n$ choisi dans la classe $E_{i}$.

2. $\left[\left[d_{l} / j_{l}\right]\right], 0 \leq j<\nu$, note, si $j_{l} \neq 0$, le développement en fraction continue du rationnel $d_{l} / j_{l}$ de longueur paire (resp. impaire) si $f_{l}(n) f_{l+1}(n)>0$ (resp. $\left.f_{l}(n) f_{l+1}(n)<0\right)$, et si $j_{l}=0$ alors $\left[\left[d_{l} / j_{l}\right]\right]$ note le vide.

3. $\beta_{\nu}=\left(\left|f_{\nu}(n)\right|-d_{\nu-1} p_{r_{\nu-1}-1}^{(\nu-1)}\right) / d_{\nu-1}^{2}$ est plus grand que 1 si $n$ assez grand, où si $j_{\nu-1} \neq 0$ et $\left[\left[d_{\nu-1} / j_{\nu-1}\right]\right]=\left[b_{1}^{(\nu-1)}, \ldots, b_{r_{\nu}}^{(\nu-1)}\right], p_{r_{\nu-1}-1}^{(\nu-1)}$ est le numérateur du rationnel $\left[b_{1}^{(\nu-1)}, \ldots, b_{r_{\nu-1}-1}^{(\nu-1)}\right]$, et si $j_{\nu-1}=0, p_{r_{\nu-1}-1}^{(\nu-1)}=0$.

4. La fonction $\beta_{\nu}$ regardée comme une fonction de $K_{X}$ est presque réduite si $\nu$ est assez grand.

5. $b_{l}(n), 0 \leq l<\nu$, sont des polynômes à coefficients rationnels à valeurs entières de degré supérieur ou égal à 1 si $l \geq 1$.

Preuve. Pour 1-3 et 5 voir [8], mais aussi [11] et [12]. Le point 4 suit du fait que $f_{\nu}$ est réduite si $\nu$ est assez grand et de 1.

On note par $r_{l}$ la longueur du développement en fraction continue du rationnel $d_{l} / j_{l}\left(r_{l}=0\right.$ si $\left.j_{l}=0\right)$ tel qu'il est défini ci-dessus.

On pose

$$
i_{-1}=-1, \quad i_{l}=l+r_{0}+r_{1}+\ldots+r_{l}, \quad l \geq 0 .
$$

On désigne par $P_{l} / Q_{l}$ (resp. $\left.\widetilde{P}_{l} / \widetilde{Q}_{l}\right)$ la $l^{\text {ième }}$ réduite de la fraction continue formelle (resp. régulière) de $f(X)$ (resp. $f(n))$. 
Enfin, on pose pour $l \geq-1, \varphi_{l+1}=P_{l}-f(X) Q_{l}$ (resp. $\widetilde{\varphi}_{l+1, n}=\widetilde{P}_{l}-$ $\left.f(n) \widetilde{Q}_{l}\right)$.

Proposition 3. Soit $f$ un élément de $K_{X}$ presque réduit. Pour tout $\nu \in \mathbb{N}^{*}$, il existe une partition de $\widetilde{\mathbb{Z}}$ en un nombre fini de classes $E_{1}, \ldots, E_{s}$ telles que pour tout $n \in E_{j}, n$ assez grand, on ait:

(i) $P_{l}(n) / Q_{l}(n)=\widetilde{P}_{i_{l}} / \widetilde{Q}_{i_{l}}, 0 \leq l<\nu$,

(ii) les nombres algébriques $\widetilde{\varphi}_{i_{l}}$ avec $0 \leq l<\nu$, sont une spécialisation des $\nu$ premiers points extrémaux de $\mathcal{M}_{X}$ dans la direction ||$_{2}$, plus précisément :

$$
\exists \lambda_{l}^{(j)} \in \mathbb{Q}^{*}, \quad \widetilde{\varphi}_{i_{l}+1}=\lambda_{l}^{(j)} \varphi_{l+1}(n), \quad-1 \leq l<\nu,
$$

(iii) les nombres algébriques $\widetilde{\varphi}_{k}, k \neq i_{l},-1 \leq l<\nu$, sont une spécialisation de fonctions algébriques de $K_{X}$ qui possèdent la propriété d'être des faces et non des points extrémaux de $\mathcal{M}_{X}$.

Preuve. Voir [8].

\section{Anneaux des stabilisateurs des modules $\mathcal{M}_{X}$ et $\mathcal{M}_{n}$}

1. Anneaux des stabilisateurs du module $\mathcal{M}_{X}$. On a posé $\mathcal{M}_{X}=\mathbb{Q}[X]+$ $f \mathbb{Q}[X]$ avec $f \in K_{X} \backslash \mathbb{Q}(X)$, module que l'on notera encore $\langle 1, f\rangle$.

Proposition 4. Soit $f \in K_{X} \backslash \mathbb{Q}(X)$. Si on écrit $f$ sous la forme

$$
f=\frac{A+B \sqrt{F}}{C}, \quad \text { avec } A, B, C \in \mathbb{Q}[X], B \neq 0, C \neq 0
$$

et les polynômes $A, B$ et $C$ premiers entre eux, et posons

$$
\begin{gathered}
C^{\prime \prime}=\operatorname{pgcd}\left(C^{2}, A C, A^{2}-B^{2} F\right), \\
C=C^{\prime \prime} C^{\prime}, \quad C^{\prime} \in \mathbb{Q}[X], \quad \Delta=C^{\prime} B \sqrt{F},
\end{gathered}
$$

alors l'anneau des stabilisateurs est

$$
\mathcal{O}_{X}=\left\langle 1, C C^{\prime} f\right\rangle=\left\langle 1, C^{\prime} B \sqrt{F}\right\rangle=\langle 1, \Delta\rangle
$$

Preuve. Voir [3], p. 152.

2. Anneaux des stabilisateurs des modules $\mathcal{M}_{n}, n \in \widetilde{\mathbb{Z}}$. Avec les notations ci-dessus et en supposant que les polynômes $A, B, C$ et $C^{\prime}$ sont à coefficients entiers ce que l'on peut faire car ceux-ci sont définis à une constante rationnelle non nulle près, on $\mathrm{a}$ : 
Proposition 5. Il existe une partition finie de $\widetilde{\mathbb{Z}}$ en un nombre fini de classes $E_{1}, \ldots, E_{s}$ et des entiers $d_{1}, \ldots, d_{s}$ tels que l'anneau des stabilisateurs du module $\mathcal{M}_{n}$ soit

$$
\mathcal{O}_{n}=\left\langle 1, \frac{C C^{\prime}}{d_{i}} f(n)\right\rangle= \begin{cases}\left\langle 1, \Delta_{i} / 2\right\rangle & \text { si } \Delta_{i}^{2} \equiv 0(\bmod 4), \\ \left\langle 1,\left(1+\Delta_{i}\right) / 2\right\rangle & \text { si } \Delta_{i}^{2} \equiv 1(\bmod 4)\end{cases}
$$

où $\Delta_{i}^{2}=\Delta^{2} / d_{i}^{2}=\left(C^{\prime 2} B^{2} / d_{i}^{2}\right) F(n)$.

Preuve. Voir [3], p. 152.

VII. Longueur de la prépériode de $f(n)$. Soit $f$ un nombre quadratique réel. On appelle longueur de la prépériode de $f$ le plus petit entier $k$ positif ou nul tel que le quotient complet $f_{k}$ du développement en fraction continue de $f$ soit réduit ( $f_{k}$ purement périodique). On la note lap $f$.

Proposition 6. Si la fonction $f(X)$ est presque réduite alors la longueur de la prépériode du nombre quadratique réel $f(n), n \in \widetilde{\mathbb{Z}}$, est bornée par 3 pour $n$ assez grand.

Pr e u ve. Rappelons la définition de " $f(X)$ presque réduit" :

$$
f(X) \text { presque réduit } \Leftrightarrow(|f(X)|>1 \text { et }|\bar{f}(X)| \leq 1)
$$

où $\bar{f}(X)$ désigne le conjugué de $f(X)$.

La première inégalité équivaut à l'inégalité $m_{0} \leq-1$, si $f(X)=$ $c_{m_{0}} X^{-m_{0}}+\ldots$; et donc $|f(n)|>1$ si on prend $n$ assez grand. De la même façon, la deuxième inégalité $|\bar{f}(X)| \leq 1$ entraîne que $|\bar{f}(n)|<C$ où $C>0$ est indépendant de $n$.

1. Supposons $f(n)>1$ pour $n$ assez grand et montrons que lap $f(n) \leq 2$ pour $n$ assez grand. En effet, il existe une partition finie $E_{1}, \ldots, E_{s}$ de $\widetilde{\mathbb{Z}}$ telle que pour tout $n \in E_{i}$,

$$
f(n)=a_{0}(n)+1 / \alpha_{1}, \quad \alpha_{1}>1,
$$

où $a_{0}(n)$ est un polynôme de degré strictement positif. On a alors

$$
\bar{f}_{1}=\frac{1}{\bar{f}(n)-a_{0}}
$$

et comme $a_{0}(n) \geq C$ pour $n$ assez grand $(\operatorname{car} f(n)>1)$ et $|\bar{f}(n)|<C$, on en déduit que $\bar{\alpha}_{1}<0$. On voit alors que le deuxième quotient complet $\alpha_{2}$ de $f(n)$, vérifie

$$
\alpha_{2}>1 \text { et }-1<\bar{\alpha}_{2}<0 .
$$

2. Supposons maintenant $f(n)<-1$ pour $n$ assez grand et montrons que dans ce cas lap $f(n) \leq 3$, pour $n$ assez grand. On a $-f(n)>1$ et donc d'après la première partie lap $(-f(n)) \leq 2$, pour $n$ assez grand. Or, $-f(n)=$ 
$b_{0}+1 / \beta_{1}, \beta_{1}>1$, soit $f(n)=-b_{0}-1+1-1 / \beta_{1}$ d'où si $f(n)=a_{0}+1 / \alpha_{1}$, $\alpha_{1}>1$, on a

$$
\alpha_{1}=\frac{\beta_{1}}{\beta_{1}-1}=1+\frac{1}{\beta_{1}-1}
$$

et donc $\alpha_{2}=\beta_{1}-1=b_{1}-1+1 / \beta_{2}, \beta_{2}>1$.

- Si $b_{1} \geq 2$ alors $\alpha_{3}=\beta_{2}$ et donc lap $f(n) \leq 3$.

- Si $b_{1}=1$ alors $0<\beta_{1}-1<1$ et $\beta_{1}-1=1 / \beta_{2}$, d'où

$$
\alpha_{1}=1+\beta_{2}=1+b_{2}+1 / \beta_{3} .
$$

Donc $\alpha_{2}=\beta_{3}$ et lap $f(n) \leq 2$.

Proposition 7. Soit $f \in K_{X}$. La longueur de la prépériode du nombre quadratique réel $f(n)$ est bornée indépendamment de l'entier $n, n \in \widetilde{\mathbb{Z}}$.

Preuve. Il résulte de la proposition 2 et de la proposition précédente qu'il existe un entier $k$ et une partition des entiers de $\widetilde{\mathbb{Z}}$ en un nombre fini de classes infinies $E_{1}, \ldots, E_{s}$ telles que pour tout $n \in E_{i}$ assez grand, on a

$$
\operatorname{lap} f(n) \leq \sum_{i=0}^{k-1}\left(L\left(d_{i} / j_{i}\right)+1\right)+3,
$$

où les entiers $d_{i}, j_{i}$ sont ceux de la proposition 2 et sont donc indépendants de l'entier $n$ choisi dans la classe $E_{i}$, et $L\left(d_{i} / j_{i}\right)=r_{i}$ est la longueur du développement en fraction continue du rationnel $d_{i} / j_{i}$ tel qu'il est défini dans la proposition 2 , si $j_{i} \neq 0$, et 0 sinon.

\section{Deux lemmes utiles}

LEMME 1. Soit $P / Q$ une fraction rationnelle à coefficients rationnels telle que $P(n) / Q(n)$ soit entier pour une infinité d'entiers $n$. Alors le polynôme $Q$ divise le polynôme $P$.

L'énoncé et la preuve de ce lemme se trouvent dans [10], chapitre VIII, problème 93.

Lemme 2. Soit $\varphi$ un élément non nul du module $\mathcal{M}_{X}$ qui n'est pas une unité formelle de $\mathcal{O}_{X}$. Alors $\varphi(n)$ n'est pas une unité de $\mathcal{O}_{n}$ pour tout entier $n \in \widetilde{\mathbb{Z}}$ sauf peut être pour un nombre fini d'entre eux.

Preuve. Posons

$$
\varphi=U+V f \quad \text { avec } U, V \in \mathbb{Q}[X] .
$$

Raisonnons par l'absurde : supposons que pour une infinité d'entiers $n$ de $\widetilde{\mathbb{Z}}$, on a $\varphi(n)$ unité de $\mathcal{O}_{n}$. On en déduit alors, d'après la proposition 5 , que $\varphi(n)$ peut s'écrire sous la forme

$$
\varphi(n)=u_{n}+v_{n} \frac{C C^{\prime}}{d} f(n)
$$


où $d_{i}$ est un entier, $C C^{\prime} \in \mathbb{Q}[X], u_{n}, v_{n} \in \mathbb{Z}$, et ceci pour une infinité de $n$ dans $\widetilde{\mathbb{Z}}$. Cette egalité peut encore s'écrire

$$
\varphi(n)=u_{n}+v_{n} C^{\prime} \frac{A+B \sqrt{F(n)}}{d} .
$$

En comparant (2) et (3), on obtient

$$
\frac{V(n) B(n)}{C(n)}=\frac{C^{\prime}(n) B(n)}{d_{i}} v_{n},
$$

d'où

$$
v_{n}=\frac{V}{C C^{\prime}} d \in \mathbb{Q}(X) \quad \text { et } \quad \frac{V(n)}{C(n) C^{\prime}(n)} d \in \mathbb{Z}
$$

pour une infinité de $n$. D'après le lemme 1 on en déduit que le polynôme $C C^{\prime}$ divise le polynôme $V$ et que donc $v_{n} \in \mathbb{Q}[X]$. On a aussi, en comparant (2) et (3)

$$
U(n)+V(n) \frac{A(n)}{C(n)}=u_{n}+\frac{C C^{\prime}}{d} A v_{n},
$$

d'où

$$
u_{n}=U+V \frac{A}{C}-\frac{C C^{\prime}}{d} A v_{n}
$$

et donc $d V(n) A(n) / C(n)$ est un entier pour une infinité de $n$. On en déduit d'après le lemme 1 que le polynôme $C$ divise le polynôme $V A$. Finalement, on a montré qu'il existe deux polynômes $S$ et $T$ à coefficients rationnels tels que

$$
u_{n}=S(n), \quad v_{n}=T(n)
$$

pour une infinité d'entiers $n$ de $\widetilde{\mathbb{Z}}$. On peut donc écrire $\varphi$ sous la forme

$$
\varphi=S_{1}+T_{1} C^{\prime} B \sqrt{F}, \quad S_{1}, T_{1} \in \mathbb{Q}[X],
$$

ce qui montre que $\varphi \in \mathcal{O}_{X}$ et comme on a aussi

$$
N \varphi=S_{1}^{2}-T_{1}^{2} C^{\prime 2} B^{2} F= \pm 1
$$

pour une infinité de $n$ dans $\widetilde{\mathbb{Z}}$ on en déduit que $\varphi$ est une unité de $\mathcal{O}_{X}$, ce qui est exclu par hypothèse.

IX. Unités relatives. Nous rappelons ici un résultat sur l'indice du sous-groupe des unités d'un ordre relativement au groupe des unités d'un autre ordre le contenant.

On considère un ordre $\mathcal{O}$ d'un corps quadratique réel. On peut l'écrire $\mathcal{O}=\mathbb{Z}[\varrho]$ où $\varrho=\sqrt{d}$ ou $\varrho=(1+\sqrt{d}) / 2$ avec $d$ entier non carré parfait.

Soit $f$ un entier. Posons $\nu(f)=\left[G: G_{f}\right]$ où $G$ (resp. $G_{f}$ ) désigne le groupe des unités de l'ordre $\mathcal{O}\left(\operatorname{resp} . \mathcal{O}_{f}=\mathbb{Z}[f \varrho]\right)$. 
Proposition 8 (H. Cohen [4]). (i) Si $f$ et $g$ sont deux entiers premiers entre eux, on a l'égalité $\nu(f g)=\operatorname{ppcm}(\nu(f), \nu(g))$.

(ii) Si $f$ est un nombre premier supérieur ou égal à 3 et soit $\alpha$ un entier supérieur ou égal à 1 . Alors $\nu\left(f^{\alpha}\right)$ divise les nombres du tableau suivant:

\begin{tabular}{|c|c|c|c|c|}
\cline { 3 - 5 } \multicolumn{1}{c|}{} & $\left(\frac{d}{f}\right)=0$ & $\left(\frac{d}{f}\right)=1$ & $\left(\frac{d}{f}\right)=-1$ \\
\hline \multirow{2}{*}{$\pi$ pair } & $f^{\alpha}$ & $\frac{f-1}{2} f^{\alpha-1}$ & $\frac{f+1}{2} f^{\alpha-1}$ \\
\hline \multirow{2}{*}{ impair } & $f \equiv 1(\bmod 4)$ & $f^{\alpha}$ & $\frac{f-1}{2} f^{\alpha-1}$ & $\frac{f+1}{2} f^{\alpha-1}$ \\
\cline { 2 - 5 } & $f \neq \equiv 1(\bmod 4)$ & & $(f-1) f^{\alpha-1}$ & $(f+1) f^{\alpha-1}$ \\
\hline
\end{tabular}

où on désigne par $\pi$ la longueur de la période du développement en fraction continue de @ et par $\left(\frac{d}{f}\right)$ le symbole de Legendre.

(iii) Si $f=2$ alors $\nu\left(2^{\alpha}\right)$ divise $2^{\alpha}$, sauf si $\varrho=(1+\sqrt{d}) / 2$ et $d \equiv 5$ $(\bmod 8)$ auquel cas $\nu\left(2^{\alpha}\right)$ divise $3 \cdot 2^{\alpha-1}$.

Corollaire. On a la majoration de l'indice

$$
\nu(f) \leq(3 / 2)^{\gamma} f
$$

où $\gamma$ désigne le nombre de facteurs premiers distincts de $f$.

X. Preuve du théorème 3. D'après la proposition 2 , on peut supposer $f$ presque réduit.

1. Le développement de $f(X)$ n'étant pas quasi-périodique, on sait d'après le théorème 2 que $\mathcal{U}_{X}=\mathbb{Q}^{*}$.

Considérons maintenant le développement en fraction continue de $f(n)$, $n \in \widetilde{\mathbb{Z}}$. La proposition 3 nous permet d'énoncer que pour tout $\nu \in \mathbb{N}^{*}$, il existe une partition finie $\left(E_{i}\right)_{1 \leq i \leq s}$ de $\widetilde{\mathbb{Z}}$ telle que pour tout $n \in E_{i}$ assez grand, les $i_{\nu-1}$ premiers points extrémaux $\widetilde{\varphi}_{k}^{(i)}\left(1 \leq k \leq i_{\nu-1}\right)$ de $\mathcal{M}_{n}$ sont la spécialisation de fonctions algébriques qui sont, soit des points extrémaux, soit des faces de $\mathcal{M}_{X}$ et ceux-ci ne sont pas des unités formelles de $\mathcal{O}_{X}$ car $\mathcal{U}_{X}=\mathbb{Q}^{*}$. On a donc

$$
\forall n \in E_{i}, \quad \widetilde{\varphi}_{k}^{(i)}=\varphi_{k}^{(i)}(n),
$$

où $\varphi_{k}^{(i)}$ est une fonction algébrique qui n'est pas une unité.

D'après le lemme 2, ces nombres quadratiques ne sont pas des unités quadratiques du corps $\mathbb{Q}(\Delta(n))$, si $n$ est assez grand, $n \in E_{i}$, et ils ne sont pas non plus congrus modulo les unités de $\mathcal{O}_{n}$ car 1 est un point extrémal de $\mathcal{M}_{n}\left(\widetilde{\varphi}_{0}^{(i)}=1\right.$, voir proposition 3$)$. On a donc

$$
\operatorname{lp} f(n) \geq i_{\nu-1}-3 \geq \nu-4,
$$

d'après la proposition 1 et la proposition 6 . 
2. Soit $\mathcal{E}(X)$ l'unité formelle de norme \pm 1 dans la direction ||$_{2}$. D'après la proposition 4 , on peut l'écrire

$$
\mathcal{E}(X)=T(X)+U(X) \Delta(X)
$$

avec $T$ et $U \in \mathbb{Q}[X]$. Posons $U=U^{*} / m$, où $U^{*} \in \mathbb{Z}[X]$ et $m \in \mathbb{N}^{*}$. Soit $n \in E_{i} \cap E$. On a

$$
\mathcal{E}(n)=T(n)+\frac{U^{*}(n) d_{i}}{m} \Delta_{i}(n)
$$

en tenant compte de l'égalité $\Delta(n)=d_{i} \Delta_{i}(n)$ (voir proposition 5 pour la définition de $d_{i}$ et $\Delta_{i}$ ).

Posons $\Delta_{i}^{2}(n)=\delta_{n}^{2} \widetilde{\Delta}_{i, n}^{2}$, avec $\delta_{n} \in \mathbb{N}$ et $\widetilde{\Delta}_{i, n}^{2}$ entier sans facteur carré.

A. Supposons $\widetilde{\Delta}_{i, n}^{2} \not \equiv 1(\bmod 4)$. Par hypothèse, on sait que $\mathcal{E}(n)$ est une unité du corps quadratique $\mathbb{Q}(\Delta(n))$ et donc $U^{*}(n) d_{i} \delta_{n} / m$ est un entier. Soit $m_{n}^{\prime}$ le p.g.c.d. de $U^{*}(n) d_{i}$ et de $m$. Posons $m_{n}^{\prime \prime}=m / m_{n}^{\prime}$; alors $m_{n}^{\prime \prime}$ divise $\delta_{n}$. Posons encore $\delta_{n}^{\prime \prime}=\delta_{n} / m_{n}^{\prime \prime} \in \mathbb{Z}$.

L'anneau des stabilisateurs du module $\mathcal{M}_{n}$ est d'après la proposition 5 , $\mathbb{Z}\left[\Delta_{i} / 2\right]$ ou $\mathbb{Z}\left[\left(1+\Delta_{i}\right) / 2\right]$. Dans les deux cas, l'anneau $\mathbb{Z}\left[\Delta_{i}\right]$ est inclus dans cet anneau de stabilisateurs. L'unité $\mathcal{E}(n)$ est par hypothèse dans $\mathbb{Z}\left[\widetilde{\Delta}_{i}\right]$ (elle est en fait dans un anneau plus petit $\mathbb{Z}\left[\delta_{n}^{\prime \prime}, \widetilde{\Delta}_{i}\right]$ ), elle n'est donc pas a priori dans l'anneau $\mathbb{Z}\left[\Delta_{i}\right]\left(\Delta_{i}=\delta_{n} \widetilde{\Delta}_{i}\right)$.

Cependant, si nous notons $G$ (resp. $G^{\prime}$ ) le groupe des unités de l'anneau $\mathbb{Z}\left[\Delta_{i}\right]$ (resp. $\mathbb{Z}\left[\delta_{n}^{\prime \prime} \widetilde{\Delta}_{i}\right]$ ), alors $\mathcal{E}^{\nu} \in \mathbb{Z}\left[\Delta_{i}\right]$ si $\nu$ désigne l'indice de $G$ dans $G^{\prime}$. Or cet indice $\nu$ est borné indépendamment de $n \in E_{i} \cap E$. En effet, d'après le corollaire de la proposition 8 , on a

$$
\nu=\nu\left(m_{n}^{\prime \prime}\right) \leq(3 / 2)^{\gamma^{\prime \prime}} m_{n}^{\prime \prime} \leq(3 / 2)^{\gamma} m
$$

où $\gamma^{\prime \prime}$ (resp. $\gamma$ ) désigne le nombre de facteurs premiers de $m_{n}^{\prime \prime}$ (resp. $m$ ). D'après les propositions 1 et 2 , on en déduit que la période du nombre quadratique $f(n), n \in E_{i} \cap E$, est bornée par

$$
\operatorname{lp} f(n) \leq \sum_{i=0}^{(\pi-1)\left[(3 / 2)^{\gamma} m\right]}\left(L\left(d_{i} / j_{i}\right)+1\right)
$$

où $\pi$ est l'entier tel que $\mathcal{E}=P_{\pi-1}-Q_{\pi-1} f$ ( $\pi$ est la longueur de la quasipériode ou son double, du développement en fraction continue formel de $f$ ).

B. Supposons $\widetilde{\Delta}_{i} \equiv 1(\bmod 4)$. On écrit dans ce cas $\mathcal{E}(n)$ sous la forme

$$
\mathcal{E}(n)=T(n)-\frac{U^{*}(n) d_{i} \delta_{n}}{m}+\frac{2 U^{*}(n) d_{i} \delta_{n}}{m}\left(\frac{1+\widetilde{\Delta}_{i}}{2}\right),
$$


ce qui permet d'en conclure que $2 U^{*}(n) d_{i} \delta_{n} / m$ est un entier. On pose

$$
m_{n}^{\prime}=\left(2 U^{*}(n) d_{i}, m\right), \quad m_{n}^{\prime \prime}=\frac{m}{m_{n}^{\prime}}, \quad \delta_{n}^{\prime \prime}=\frac{\delta_{n}}{m_{n}^{\prime \prime}} .
$$

L'anneau des stabilisateurs du module $\mathcal{M}_{n}$ est, d'après la proposition 5 , $\mathbb{Z}\left[\Delta_{i} / 2\right]$ ou $\mathbb{Z}\left[\left(1+\Delta_{i}\right) / 2\right]$. Dans les deux cas, l'anneau $\mathbb{Z}\left[\Delta_{i}\right]$ est inclus dans l'anneau des stabilisateurs et donc si $\mathcal{E}^{\nu} \in \mathbb{Z}\left[\Delta_{i}\right]$, on pourra avoir un renseignement sur la longueur de la période de $f(n)$. Plus précisément, avec les notations du paragraphe IX, si on prend $\nu=\nu\left(2 m_{n}^{\prime \prime}\right)$ alors $\mathcal{E}^{\nu} \in \mathbb{Z}\left[\Delta_{i}\right]$ or $\nu\left(2 m_{n}^{\prime \prime}\right) \leq 2(3 / 2)^{\gamma^{\prime \prime}} m_{n}^{\prime \prime} \leq 2(3 / 2)^{\gamma} m$ (corollaire de la proposition 8 ), d'où

$$
\operatorname{lp} f(n) \leq \sum_{i=0}^{(\pi-1)\left[(3 / 2)^{\gamma} 2 m\right]}\left(L\left(d_{i} / j_{i}\right)+1\right), \quad \forall n \in E_{i} \cap E,
$$

où $\pi$ est l'entier tel que $\mathcal{E}=P_{\pi-1}-Q_{\pi-1} f$. Ceci finit la preuve de (i).

Montrons maintenant (ii). Soit $n \in \widetilde{\mathbb{Z}}-E$. Comme au point 1 , les $k(\pi-1)-$ premiers points extrémaux du module $\mathbb{Z}+\mathbb{Z} f(n)$ ne sont pas des unités du corps quadratique $\mathbb{Q}\left(\Delta_{n}\right)$ (et donc ne sont pas des unités de l'anneau des stabilisateurs de ce module) pour $n$ assez grand sauf peut-être ceux d'indice $l(\pi-1)$, pour $l$ compris entre 1 et $k$. Montrons qu'il en est de même pour celles-ci. En effet, supposons le contraire : $\mathrm{Si} \varphi_{l(\pi-1)}(n)$ était une unité du corps quadratique pour un $l$ avec $1 \leq l \leq k$ alors on en déduirait que $\varphi_{\pi-1}(n)$ en serait une, ce qui est contraire à l'hypothèse faite sur $n$. Par suite, $\operatorname{lp} \alpha(n) \geq k(\pi-1)$.

\section{Références}

[1] W. W. Adams and M. J. Razar, Multiples of points on elliptic curves and continued fractions, Proc. London Math. Soc. 41 (1980), 481-498.

[2] T. G. Berry, On periodicity of continued fractions in hyperelliptic function fields, Arch. Math. (Basel) 55 (1990), 259-266.

[3] Z. I. Borevitch et I. R. Chafarevitch, Théorie des nombres, Gauthier-Villars, 1967.

[4] H. Cohen, Multiplication par un entier d'une fraction continue périodique, Acta Arith. 26 (1974), 129-148.

[5] Y. Hellegouarch, D. L. McQuillan et R. Paysant-Le Roux, Unités de certains sous-anneaux des corps de fonctions algébriques, ibid. 48 (1987), 9-47.

[6] R. Paysant-Le Roux, Périodicité des fractions continues dans un corps de fonctions hyperelliptiques, Arch. Math. (Basel) 61 (1993), 46-58.

[7] —, Calibre d'un corps arithmétique. Unités, Thèse, 1987.

[8] R. Paysant-Le Roux, Y. Hellegouarch et M. L. Gaunet, Sur certaines fractions continues non standard, C. R. Acad. Sci. Paris Sér. I 316 (1993), 763-768.

[9] O. Perron, Die Lehre von den Kettenbrüchen, dritte Auflage, Stuttgart, 1954.

[10] G. Pólya und G. Szegö, Aufgaben und Lehrsätze aus der Analysis, II Band, Springer, 1964. 
[11] A. Schinzel, On some problems of the arithmetical theory of continued fractions, I, Acta Arith. 6 (1961), 393-413; II, ibid. 7 (1962), 287-298; Corrigendum, ibid. 47 (1986), 295.

[12] H. Schmidt, Zur Approximation und Kettenbruchentwicklung quadratischer Zahlen, Math. Z. 52 (1950), 168-192.

U.F.R. SCIENCE

UNIVERSITÉ DE CAEN

ESPLANADE DE LA PAIX

14000 CAEN CEDEX, FRANCE 